\title{
Dissolution Dynamic Nuclear Polarization-Enhanced Zero- to Ultralow-Field Nuclear Magnetic Resonance
}

\author{
Román Picazo-Frutos,,${ }^{1,2, *}$ Quentin Stern, ${ }^{3, \dagger}$ John W. Blanchard, ${ }^{1}$ Olivier Cala, ${ }^{3}$ Morgan Ceillier,${ }^{3}$ \\ Samuel F. Cousin, ${ }^{3}$ James Eills, ${ }^{1,2}$ Stuart J. Elliott, ${ }^{3,4}$ Sami Jannin, ${ }^{3}$ and Dmitry Budker ${ }^{1,2,5}$ \\ ${ }^{1}$ Helmholtz Institute Mainz, (Germany), GSI Helmholtzzentrum für Schwerionenforschung, 64278 Darmstadt (Germany) \\ ${ }^{2}$ Johannes Gutenberg University, 55099 Mainz (Germany) \\ ${ }^{3}$ Université de Lyon, CNRS, Université Claude Bernard Lyon 1, ENS de Lyon, \\ Institut des Sciences Analytiques, UMR 5280, 69100 Villeurbanne, France \\ ${ }^{4}$ Department of Chemistry, University of Liverpool, Liverpool L69 7ZD, United Kingdom \\ ${ }^{5}$ Department of Physics, University of California, Berkeley, CA 94720-7300 USA
}

(Dated: February 15, 2022)

\begin{abstract}
Zero- to ultralow- field nuclear magnetic resonance is a modality of magnetic resonance experiment which does not require strong superconducting magnets. Contrary to conventional high-field nuclear magnetic resonance, it has the advantage of allowing high resolution detection of nuclear magnetism through metal as well as within heterogeneous media. To achieve high sensitivity, it is common to couple zero-field nuclear magnetic resonance with hyperpolarization techniques. To date, the most common technique is parahydrogen induced polarization, which is only compatible with a small number of compounds. In this article, we establish dissolution dynamic nuclear polarization as a versatile method to enhance signals in zero-field nuclear magnetic resonance experiments on virtually all small molecules with $>1 \mathrm{~s}$ relaxation times. We show as first examples $J$-spectra of hyperpolarized $\left[{ }^{13} \mathrm{C}\right]$ sodium formate, $\left[1-{ }^{13} \mathrm{C}\right]$ glycine and $\left[2-{ }^{13} \mathrm{C}\right]$ sodium acetate. We find signal enhancements of up to 11000 compared with thermal prepolarization in a $2 \mathrm{~T}$ permanent magnet. To increase the signal in future experiments, we investigate the relaxation effects of the TEMPOL radicals used for the hyperpolarization process at zero- and untralow-field.
\end{abstract}

\section{INTRODUCTION}

Nuclear magnetic resonance (NMR) is a wellestablished experimental method with applications in biology, chemical analysis, medical imaging, and fundamental research. Apart from the low intrinsic sensitivity in NMR, this technique suffers from two main drawbacks: First, the inability to observe signals through conductive material due to the low skin depth at high frequencies, with an exception of a few special cases, e.g. where the metal enclosure itself could be used as a resonator in a tuned circuit [1]. Second, the significant spectral broadening in heterogeneous materials due to absolute field inhomogeneity.

Recent years have seen the development of a new experimental method that overcomes these drawbacks by not requiring the use of strong magnets: zero- to ultralow-field NMR (ZULF NMR) [2]. Chemical shifts vanish in the absence of magnetic fields, however structural elucidation and chemical fingerprint can be obtained via the dominant spin-spin $J$-interaction [3]. ZULF NMR enables observation of signals through conductive material due to the $\nu^{-1 / 2}$ frequency dependence of the skin-depth in metal; the skin depth of common metals is in the $\mu \mathrm{m}$ range for radio frequencies (rf) but increases to $\mathrm{mm}$ for audio frequencies [4]. Furthermore,

\footnotetext{
* ropicazo@uni-mainz.de; These authors have contributed equally to this work

† quentin.stern@protonmail.com; These authors have contributed equally to this work
}

ZULF NMR is immune to magnetic-field inhomogeneities induced by heterogeneous materials, resulting in narrow lines in the order of tens of $\mathrm{mHz}$ [5]. ZULF NMR also offers the possibility of studying interactions invisible in conventional high-field NMR experiments, mainly limited to secular terms with respect to the high external magnetic field. Because of this, there has been an increasing interest in using zero-field NMR for research outside the Standard Model of physics, such as searching for exotic spin-spin interactions and dark matter $[6,7]$.

Despite the many advantages of ZULF NMR, its main limitation is low sensitivity: at zero field there is negligible spin magnetization, and therefore an external source of spin polarization is required. In early ZULF-NMR experiments, the sample was prepolarized in a magnetic field where spins reach thermal equilibrium polarization, obeying the Boltzmann distribution of state populations, after which the sample was brought to zero field for detection $[8,9]$. The disadvantage of this method is the low-polarization levels that can be obtained, on the order of $10^{-6}$ at tesla-level fields.

An alternative approach is hyperpolarization: experimental techniques that yield nuclear spin polarization levels several orders of magnitude higher than thermal equilibrium resulting in enhanced NMR signals [10, 11]. Hyperpolarization has been particularly useful in combination with benchtop NMR devices in which thermal equilibrium polarization can be prohibitively low, since they operate at fields of no more than a few tesla [12]. Importantly, the polarization is no longer governed by the detection magnetic-field strength, but rather the hyperpolarization method and details of the hyperpolarization 
procedure. Parahydrogen induced polarization (PHIP) $[13,14]$ and spin-exchange optical pumping (SEOP) [1518] have been used to hyperpolarize samples for zeroand moderate-field NMR experiments, but PHIP relies on specific chemical reactions with hydrogen gas, and SEOP is limited to polarizing noble gas atoms.

Dissolution dynamic nuclear polarization ( $d \mathrm{DNP})[19]$ is a more general technique, allowing one to polarize a broad variety of molecules to high polarization levels. Indeed, $d$ DNP has established itself as the hyperpolarization method of choice for polarizing a wide range of small molecules [20,21]. This is achieved by bringing the sample to cryogenic temperatures (typically $1.2 \mathrm{~K}$ ) commonly at fields in the $3.3-7 \mathrm{~T}$ range in the presence of unpaired electrons, whose polarization is near unity under these conditions. The electron polarization is transferred to the nuclear spins via microwave irradiation after which the frozen sample is dissolved using a hot solvent and transferred to an NMR spectrometer or MRI scanner for use of the hyperpolarized solution in the liquid state. This technique has the advantage of broad applicability, with polarization levels of tens of percent regularly achieved [22]. A first demonstration of $d \mathrm{DNP}$ coupled with ZULF-NMR detection was recently presented [23], where $\left[1-{ }^{13} \mathrm{C}\right]$ pyruvic acid was hyperpolarized and detected with a portable zero-field spectrometer.

In this work we show an order of magnitude improvement in sensitivity, and demonstrate hyperpolarization and zero-field detection of multi-component sample mixtures. We demonstrate fine experimental control over the process, integrating the hyperpolarization apparatus with the ZULF spectrometer. We obtain spin polarization and therefore sensitivity enhancements of 11000 with respect to a conventional ZULF-NMR experiment with sample prepolarization at $2 \mathrm{~T}$. We evaluate paramagnetic relaxation induced by the DNP polarizing agent as a possible reason for the obtained signal enhancement of 11000 while up to $>50 \mathrm{k}$ could be expected theoretically. We discuss possible improvements of $d$ DNP-ZULF NMR based on recently reported methods such as fast dissolution, transfer, and sample injection[24], as well as the use of filterable polarizing matrices to reduce paramagnetic relaxation [25].

\section{THEORY}

We calculate the signal enhancement of $d$ DNP-ZULF experiments with respect to thermal prepolarization for a heteronuclear pair of $J$-coupled spins-1/2 with positive gyromagnetic ratios. The relevant nuclear spin Hamiltonian $\mathcal{H}$ for a pair of $J$-coupled spins $\mathrm{I}$ and $\mathrm{S}$ has the form:

$$
\mathcal{H}=\omega_{\mathrm{I}}^{0} I_{z}+\omega_{\mathrm{S}}^{0} S_{z}+2 \pi J_{\mathrm{IS}} \mathbf{I} \cdot \mathbf{S},
$$

where $\omega_{\mathrm{I}}^{0}$ and $\omega_{\mathrm{S}}^{0}$ are the nuclear Larmor frequencies of spins I and $\mathrm{S}$ in $\mathrm{rad} \mathrm{s}^{-1}$ respectively, and $J_{\mathrm{IS}}$ is the scalar coupling constant between spins I and $\mathrm{S}$ in
$\mathrm{Hz}$. At high magnetic field, the eigenstates of the 2spin- $1 / 2$ system are given by the Zeeman basis $\mathcal{B}_{\mathcal{Z}}=$ $\{|\alpha \alpha\rangle,|\alpha \beta\rangle,|\beta \alpha\rangle,|\beta \beta\rangle\}$. In the absence of magnetic field, the Zeeman terms vanish and so the zero-field Hamiltonian $\mathcal{H}_{\text {ZF }}$ consists only of the $J$-coupling part:

$$
\mathcal{H}_{\mathrm{ZF}}=2 \pi J_{\mathrm{IS}} \mathbf{I} \cdot \mathbf{S} .
$$

The eigenbasis at zero field is the singlet-triplet basis $\mathcal{B}_{\mathcal{S T}}$ :

$$
\begin{aligned}
\left|T_{+1}\right\rangle & =|\alpha \alpha\rangle, \\
\left|S_{0}\right\rangle & =\frac{1}{\sqrt{2}}(|\alpha \beta\rangle-|\beta \alpha\rangle), \\
\left|T_{0}\right\rangle & =\frac{1}{\sqrt{2}}(|\alpha \beta\rangle+|\beta \alpha\rangle), \\
\left|T_{-1}\right\rangle & =|\beta \beta\rangle .
\end{aligned}
$$

We represent the state of the system using the density matrix formalism: if $P_{\mathrm{I}}$ and $P_{\mathrm{S}}$ are the polarizations of spin I and $\mathrm{S}$ before detection respectively, the density matrix $\rho_{0}$ representing the spin system is given by the Kronecker product:

$$
\rho_{0}=\rho_{I} \otimes \rho_{S}=\left(\frac{\mathbb{1}}{2}+P_{\mathrm{I}} I_{z}\right) \otimes\left(\frac{\mathbb{1}}{2}+P_{\mathrm{S}} S_{z}\right),
$$

where $\mathbb{1}$ is the unity operator. In order to obtain a signal, coherences between eigenstates must be excited. In our experiments, we rapidly switch off the field which projects the Zeeman states onto the singlet-triplet basis. The $|\alpha \alpha\rangle$ and $|\beta \beta\rangle$ states remain eigenstates so they do not evolve under $\mathcal{H}_{\mathrm{ZF}}$. The $|\alpha \beta\rangle$ and $|\beta \alpha\rangle$ states are no longer eigenstates of $\mathcal{H}_{\mathrm{ZF}}$ but are superpositions of the $\left|S_{0}\right\rangle$ and $\left|T_{0}\right\rangle$ states. Therefore, the sudden field drop induces a coherence between $\left|S_{0}\right\rangle$ and $\left|T_{0}\right\rangle$. The amplitude of this transition is given by:

$$
p_{S_{0} \rightarrow T_{0}}=\operatorname{Tr}\left\{\left|S_{0}\right\rangle\left\langle T_{0}\right| \rho_{0}\right\}=\frac{1}{4}\left(P_{\mathrm{I}}-P_{\mathrm{S}}\right) .
$$

The magnetometer is sensitive to magnetization along the $z$-axis:

$$
S \propto M_{z} \propto\left|P_{\mathrm{I}}-P_{\mathrm{S}}\right|,
$$

where $S$ is the oscillating signal recorded by the magnetometer. Note that this result is only valid if the signs of the gyromagnetic ratios of I and S are equal. We define the enhancement as the ratio of the signal recorded with $d \mathrm{DNP}$ hyperpolarization $S^{\mathrm{HP}}$ and the signal recorded with thermal prepolarization $S^{\text {th }}$ :

$$
\varepsilon=\frac{S^{\mathrm{HP}}}{S^{\text {th }}}=\left|\frac{P_{\mathrm{I}}^{\mathrm{HP}}-P_{\mathrm{S}}^{\mathrm{HP}}}{P_{\mathrm{I}}^{\mathrm{th}}-P_{\mathrm{S}}^{\text {th }}}\right|,
$$

where $P_{\mathrm{I}}^{\mathrm{HP}}, P_{\mathrm{S}}^{\mathrm{HP}}, P_{\mathrm{I}}^{\text {th }}, P_{\mathrm{S}}^{\text {th }}$ are the polarizations of spins I and S hyperpolarized by $d \mathrm{DNP}$ and thermally prepolarized at a field $B_{p}$, respectively. The signal $S^{\text {th }}$ cannot be 
directly recorded on a sample in a $d \mathrm{DNP}$ experiment because, after dissolution, the analyte concentration is typically on the order of $25-50 \mathrm{mM}$, too low to be efficiently detected at zero field with thermal prepolarization. To circumvent this, we acquire the signal on a reference sample $S_{\text {ref }}^{\text {th }}$ with higher analyte concentration $(>1 \mathrm{M})$ prepolarized at $2 \mathrm{~T}$. We define $\tilde{\varepsilon}$ as the ratio of the integrals of the hyperpolarized signal $S^{\mathrm{HP}}$ and the thermal reference, with the following equation relating the two enhancement definitions:

$$
\begin{aligned}
& \tilde{\varepsilon}=\frac{S^{\mathrm{HP}}}{S_{\mathrm{ref}}^{\mathrm{th}}}, \\
& \varepsilon=\tilde{\varepsilon} \frac{C_{\mathrm{ref}}^{\mathrm{th}}}{C^{\mathrm{HP}}},
\end{aligned}
$$

where $C_{\text {ref }}^{\text {th }}$ and $C^{\mathrm{HP}}$ are the concentrations of the reference sample and of that resulting from the $d \mathrm{DNP}$ experiment, respectively. $\tilde{\varepsilon}$ is the measurable in our experiment and the calculated $\varepsilon$ serves as a tool to understand the polarization enhancement.

The enhancement factor defined by Eq. 10 is equivalent to that commonly used in high field $d \mathrm{DNP}[22]$. It corresponds to the ratio between the signals measured with and without hyperpolarization on the same sample and in the same conditions.

\section{METHODS AND EXPERIMENTAL SETUP}

\section{A. Samples}

All chemicals were purchased from Sigma-Aldrich. For thermal prepolarization experiments, we used $5.2 \mathrm{M}$ $\left[{ }^{13} \mathrm{C}\right]$ sodium formate dissolved in $\mathrm{D}_{2} \mathrm{O}$, degassed by $\mathrm{N}_{2^{-}}$ bubbling for $10 \mathrm{~min}$ to remove paramagnetic oxygen, which would contribute to nuclear spin relaxation.

For $d$ DNP experiments, we used $50 \mathrm{mM}$ of 4-hydroxy2,2,6,6-tetramethylpiperidin-1-oxyl (TEMPOL) as the polarizing agent (PA) [20]. We prepared two sample mixtures: the first mixture contained $1.5 \mathrm{M}\left[2-{ }^{13} \mathrm{C}\right]$ sodium acetate and $1.5 \mathrm{M}\left[{ }^{13} \mathrm{C}\right]$ sodium formate. We refer to this mixture as HP1. The second mixture, HP2, contained $0.76 \mathrm{M}\left[1-{ }^{13} \mathrm{C}\right]$ sodium pyruvate, $0.80 \mathrm{M}\left[{ }^{13} \mathrm{C}\right]$ sodium formate, and $0.85 \mathrm{M}\left[1-{ }^{13} \mathrm{C}\right]$ glycine. Both samples were dissolved in $\mathrm{H}_{2} \mathrm{O} / \mathrm{D}_{2} \mathrm{O} / \mathrm{D}_{8}$-glycerol $1: 3: 6(v / v / v)$, which ensures good dispersion of the radical upon freezing. This mixture is known as "DNP juice" [20, 26, 27].

\section{B. $d \mathrm{DNP}$ hyperpolarization experiments}

The experimental sequence consists of three steps: hyperpolarization by low-temperature DNP with cross polarization (CP), sample dissolution and transfer from the polarizer to the ZULF spectrometer, and liquid-state detection at zero field. The experimental sequence and setup are summarized in Fig. 1.

\section{Solid-state DNP}

A prototype $d$ DNP polarizer "Alpha Polarizer" developed by Bruker Biopspin ${ }^{\circledR}$ was used to hyperpolarize the samples. $100 \mu \mathrm{l}$ of HP1 or HP2 was first frozen by freezing individual $10 \mu \mathrm{l}$ drops in liquid $\mathrm{N}_{2}$. The resulting beads were transferred into a PEEK sample holder. In addition to the analytes, the sample holder was also loaded with 10 beads of $10 \mu \mathrm{l} 3 \mathrm{M}$ ascorbic acid dissolved in $\mathrm{D}_{2} \mathrm{O}$, as well as an additional $30 \mathrm{mM}$ of sodium ascorbate to the dissolution heating module containing the hot solvent, in order to reduce the polarization loss due to paramagnetic relaxation [27]. The sample holder was placed in the liquid helium bath of the cryostat and the temperature was lowered to $1.2 \mathrm{~K}$ by pumping the cryostat to $0.65 \mathrm{mbar}$.

The electron polarization was transferred to the ${ }^{1} \mathrm{H}$ nuclear spins by microwave irradiation $(\mu \mathrm{w})$ with a frequency centered at $192.65 \mathrm{GHz}$ [28] and modulated at a rate of $500 \mathrm{~Hz}$ over a bandwidth of $160 \mathrm{MHz}$.

The polarization was transferred from ${ }^{1} \mathrm{H}$ to ${ }^{13} \mathrm{C}$ spins every $4 \mathrm{~min}$ using microwave-gated $\mathrm{CP}$ as described in references [29-32]. The polarization of the ${ }^{13} \mathrm{C}$ spins during this process was monitored using $5^{\circ} \mathrm{rf}$ pulses applied every $30 \mathrm{~s}$. According to Eq. 9, the signal is maximal if the respective polarizations of ${ }^{1} \mathrm{H}$ and ${ }^{13} \mathrm{C}$ spins have opposite signs. Therefore, once the polarization plateaued, the microwave source was turned off and the ${ }^{13} \mathrm{C}$ polarization was inverted using an adiabatic frequency swept inversion pulse (chirp pulse) of $1 \mathrm{~ms}$ duration and $80 \mathrm{kHz}$ bandwidth. At this point, the ${ }^{1} \mathrm{H}$ and ${ }^{13} \mathrm{C}$ spins had positive and negative polarization, respectively, which maximizes the signal according to Eq. 9.

\section{Dissolution and transfer}

The dissolution step was performed immediately so as to minimize relaxation in the solid state. $5 \mathrm{ml}$ of $\mathrm{D}_{2} \mathrm{O}$ with $30 \mathrm{mM}$ ascorbic acid was loaded into a heating module, pressurized to 6 bar with $\mathrm{He}$ gas and subsequently heated to $180^{\circ} \mathrm{C}$ corresponding to a pressure of 9 bar. The dissolution step consisted of a series of programmed events triggered by the operator. The pressurized hot solvent was injected onto the sample and pushed with $\mathrm{He}$ gas at 9 bar through a KelF capillary to the ZULF spectrometer. The capillary was inside a solenoid maintaining a field of $4 \mathrm{mT}$ along the transfer, from the polarizer to the magnet used for thermal prepolarization experiments. The flow of He gas was stopped after a transfer time $t_{\text {trans }}=2 \mathrm{~s}$.

\section{Injection and $Z U L F$ detection}

We used a home-built ZULF spectrometer for signal detection which is a modified version of the setup described in reference [33]. Using a four-layer $\mu$-metal shield (MS-1, Twinleaf LCC) and additional shimming 
(a)

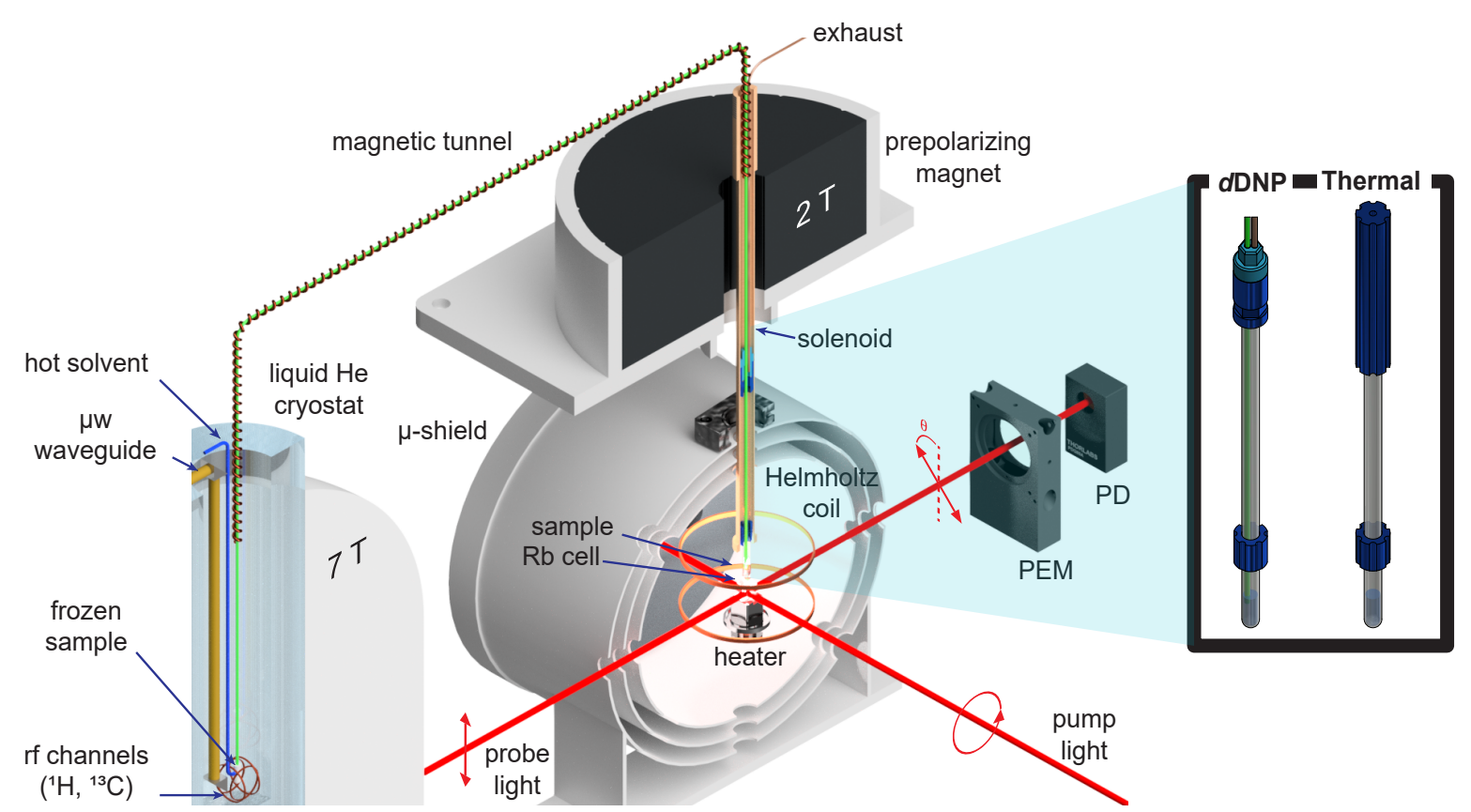

(b)

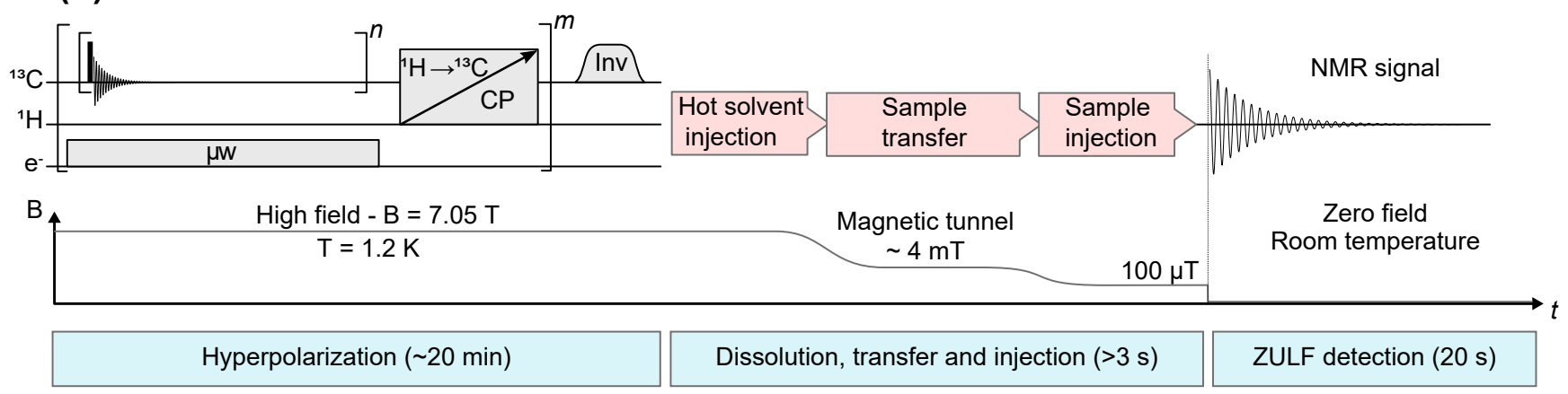

FIG. 1. (a) Schematic of the experimental apparatus. The sample is hyperpolarized by $d$ DNP and is transferred to the ZULF spectrometer for detection. The spectrometer is triggered upon sample arrival and the magnetic field is suddenly switched off prior to signal acquisition. A magnetic tunnel of $4 \mathrm{mT}$ maintains polarization during sample transfer. The insert shows the sample assemblies for the thermal and $d \mathrm{DNP}$ experiments. $\mathrm{PEM}=$ Photoelastic modulator, $\mathrm{PD}=$ photodiode. Note that the $2 \mathrm{~T}$ magnet is built into the setup for prepolarization experiments, but is not part of the $d \mathrm{DNP}$ experiment and is ignored in the experimental sequence. More details can be found in the main text. (b) Experimental sequence from hyperpolarization in the solid state at $1.2 \mathrm{~K}$ and $7.05 \mathrm{~T}$ to detection in the liquid state at zero magnetic field. The gray blocks " $\mu \mathrm{w}$ ", "CP", and "Inv" represent $\mu \mathrm{w}$ irradiation, polarization transfer from ${ }^{1} \mathrm{H}$ to ${ }^{13} \mathrm{C}$ by cross polarization and adiabatic inversion pulse acting on ${ }^{13} \mathrm{C}$ spins, respectively. The numbers $n$ and $m$ correspond to the number of ${ }^{13} \mathrm{C}$ detection blocks between each $\mathrm{CP}$ contact and the total number of $\mathrm{CP}$ contacts in the hyperpolarization scheme, respectively. Typically $n=6$ and $m \geq 6$.

coils, the residual field at the sample location was brought down to sub-nT levels. At the center of the detection region, a 3D-printed holder accommodated a standard NMR tube and a Helmholtz-coil pair along the sensitive axis. A magnetic tunnel maintaining a field of $4 \mathrm{mT}$ was connected to another guiding solenoid used to shuttle the sample to the detection region.

At the beginning of the dissolution sequence, the ZULF spectrometer was triggered and it immediately switched on the guiding solenoid and Helmholtz-coil pair, both providing a $100 \mu \mathrm{T}$ field. After the transfer, the hyperpolarized sample reached the NMR tube waiting in the detection region which has a PEEK assembly containing input and output capillaries for injection and exhaust. $4 \mathrm{~s}$ after receiving the trigger, the guiding solenoid was switched off. After an additional delay of $100 \mathrm{~ms}$ allowing the solenoid field to decay, the Helmholtz coil was switched off within $10 \mu \mathrm{s}$, bringing the spins nonadiabatically to zero field to generate an observable signal. As mentioned above, a flow of He gas pushed the 
solution from the polarizer during $t_{\text {trans }}=2 \mathrm{~s}$. The detection was performed $4.1 \mathrm{~s}$ after the ZULF spectrometer received the trigger from the $d \mathrm{DNP}$ system, meaning that the solution was left at $100 \mu \mathrm{T}$ during a settling time $t_{\text {settle }}=2.1 \mathrm{~s}$. This delay allowed the pressure of the $\mathrm{He}$ propeller gas to equilibrate with atmospheric pressure for the sample to settle.

The signal was obtained via optical rotation of linearly polarized light off-resonant to the $\mathrm{D} 2$ transition of ${ }^{87} \mathrm{Rb}$ and circularly polarized with a laser pump beam tuned to the D1 transition of ${ }^{87} \mathrm{Rb}$ in a cross-beam laser configuration [34]. The laser beams cross in the center of a $\mathrm{Rb}$ vapor cell (500 torr $\mathrm{N}_{2}$ buffer gas) with outer dimensions of $8 \times 8 \times 10 \mathrm{~mm}^{3}$ and $1 \mathrm{~mm}$-thickness walls (Twinleaf LCC). A photoelastic modulator (II/FS42A PEM, Acal BFi Germany GmbH) was used to modulate the signal at frequencies of $50 \mathrm{kHz}$ and a lock-in amplifier (SRS 830, Standard Research Systems) demodulated the signal back to audio frequencies. The demodulated signal was then acquired with an acquisition card (NI9239, National Instruments, Austin, USA) and magneticfield pulses and digital triggers were provided by output modules (NI-9263 and NI-9401, National Instruments, Austin,USA).

In all experiments, the atomic magnetometer had a sensitivity of $40-80 \mathrm{fT}_{\mathrm{rms}} / \sqrt{\mathrm{Hz}}$ in the frequency range 1$300 \mathrm{~Hz}$. It is worth noting that the PEEK assembly of the sample allows the user to keep the guiding solenoid and prepolarizing magnet of the thermal experiment, which improves the duty cycle and polarization maintenance, respectively.

\section{Analyte-concentration measurement}

After the measurement of ZULF spectra of hyperpolarized samples, the concentration of formate ions was measured by recording the ${ }^{1} \mathrm{H}$ NMR signal at $1.88 \mathrm{~T}$ on a Bruker ${ }^{\circledR}$ Fourier 80 benchtop spectrometer.

\section{Thermal-polarization experiment}

In order to obtain a thermal-reference comparison for our hyperpolarization experiments, we pneumatically shuttled the sample within $0.5 \mathrm{~s}$ using a vacuum pump from the center of a $2 \mathrm{~T}$ prepolarizing Halbach magnet to the detection region. Similar to the $d \mathrm{DNP}$ experiments, once the sample reached the center of the zero-field chamber, the guiding fields were rapidly switched off and the signal was detected. The NMR tube was assembled with PEEK pieces for efficient translation along the guiding solenoid. This is shown in the insert of Fig. 1.

\section{RESULTS}

\section{A. $d \mathrm{DNP}$ experiments}

The ZULF spectra of hyperpolarized samples HP1 and HP2 are shown in Fig. 2. A thermal reference experiment with prepolarization at $2 \mathrm{~T}$ averaged over sixteen scans of a sample of $5.2 \mathrm{M}\left[{ }^{13} \mathrm{C}\right]$ sodium formate in $\mathrm{D}_{2} \mathrm{O}$ is shown for comparison (black curve). The thermal reference spectrum is scaled and offset for better visualization. The noise peaks arising from the power line at $50 \mathrm{~Hz}$ and overtones (commonly found in ZULF NMR [33]) are grayed out, and probe-laser noise peaks are depicted with asterisks.

To understand the peaks in the ZULF spectrum it is useful to consider the Pople notation [35]. The molecules of interest can be modeled as an $\mathrm{XA}_{n}$ system, where magnetically equivalent spins $\mathrm{A}_{n}$ are equally coupled to a heteronuclear spin $\mathrm{X}$. In the ZULF regime, the $J$-coupling interaction is dominant and causes observable transitions at integer and half-integer multiples of the $J$-coupling constant. In this work, we studied $\left[{ }^{13} \mathrm{C}\right]$ sodium formate, $\left[1-{ }^{13} \mathrm{C}\right]$ glycine, and $\left[2-{ }^{13} \mathrm{C}\right]$ acetate, which correspond to $\mathrm{XA}, \mathrm{XA}_{2}$, and $\mathrm{XA}_{3}$ spin systems, respectively. These systems have observable transitions at $J_{\mathrm{XA}}, 3 / 2 \times J_{\mathrm{XA}}$, and $J_{\mathrm{XA}}$ and $2 \times J_{\mathrm{XA}}$, respectively [36, 37].

The ZULF spectrum of the hyperpolarized sample HP1 features peaks at ${ }^{1} J_{\mathrm{CH}}=194.7 \mathrm{~Hz}$ for formate and two peaks at ${ }^{1} J_{\mathrm{CH}}=127.3 \mathrm{~Hz}$ and $2 \times{ }^{1} J_{\mathrm{CH}}$ for acetate (see Fig. 2b). Compared to prepolarization at $2 \mathrm{~T}$, the sodium formate signal enhancement translates into a spin polarization enhancement of $\varepsilon=5000$. Indeed, the peak shows a signal enhancement $\tilde{\varepsilon}=44$ with a concentration of formate in the dissolved sample of HP1 determined to be $45 \mathrm{mM}$ by high field NMR, while the reference sample was at a higher concentration of $5.2 \mathrm{M}$.

In the ZULF spectrum of the hyperpolarized sample $\mathrm{HP} 2$, peaks can be clearly identified at ${ }^{1} J_{\mathrm{CH}}=194.7 \mathrm{~Hz}$ for formate and one peak at $3 / 2 \times{ }^{2} J_{\mathrm{CH}}=8.0 \mathrm{~Hz}$ for glycine (see Fig. 2c). The expected signals of pyruvate at ${ }^{3} J_{\mathrm{CH}}=1.3 \mathrm{~Hz}$ and $2 \times{ }^{3} J_{\mathrm{CH}}=2.6 \mathrm{~Hz}$ are not visible in the spectrum. The formate concentration in the dissolved sample was determined to be $38 \mathrm{mM}$. Compared to prepolarization at $2 \mathrm{~T}$, the sodium formate peak shows a spin polarization enhancement of $\varepsilon=11000$ (concentration ratio of 137 and signal enhancement of $\tilde{\varepsilon}=83$ ).

To estimate the ${ }^{1} \mathrm{H}$ and ${ }^{13} \mathrm{C}$ polarization of the analyte that could be expected from our $d \mathrm{DNP}$ experiments, we performed a similar $d \mathrm{DNP}$ experiment on $\left[1-{ }^{13} \mathrm{C}\right]$ sodium acetate injected into a Bruker Fourier 80 benchtop spectrometer operating at $1.88 \mathrm{~T}$. We found ${ }^{1} \mathrm{H}$ and ${ }^{13} \mathrm{C}$ polarizations were $6 \%$ and $20 \%$, respectively (see Fig. 3a). In ZULF experiments, these polarization levels would translate into an enhancement of $\varepsilon=51000$ with respect to prepolarization at $2 \mathrm{~T}$ according to Eq. 10 (provided the ${ }^{13} \mathrm{C}$ spins are efficiently inverted prior to dissolution to yield $P\left[{ }^{13} \mathrm{C}\right]=-20 \%$ ). Such enhancement can be expected in the $d$ DNP-ZULF experiment. However, our 
(a)
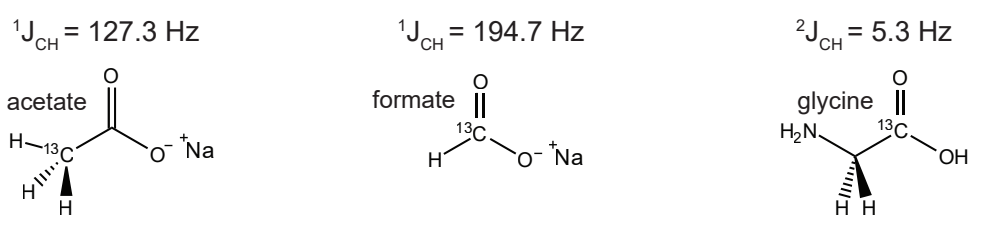

(b)
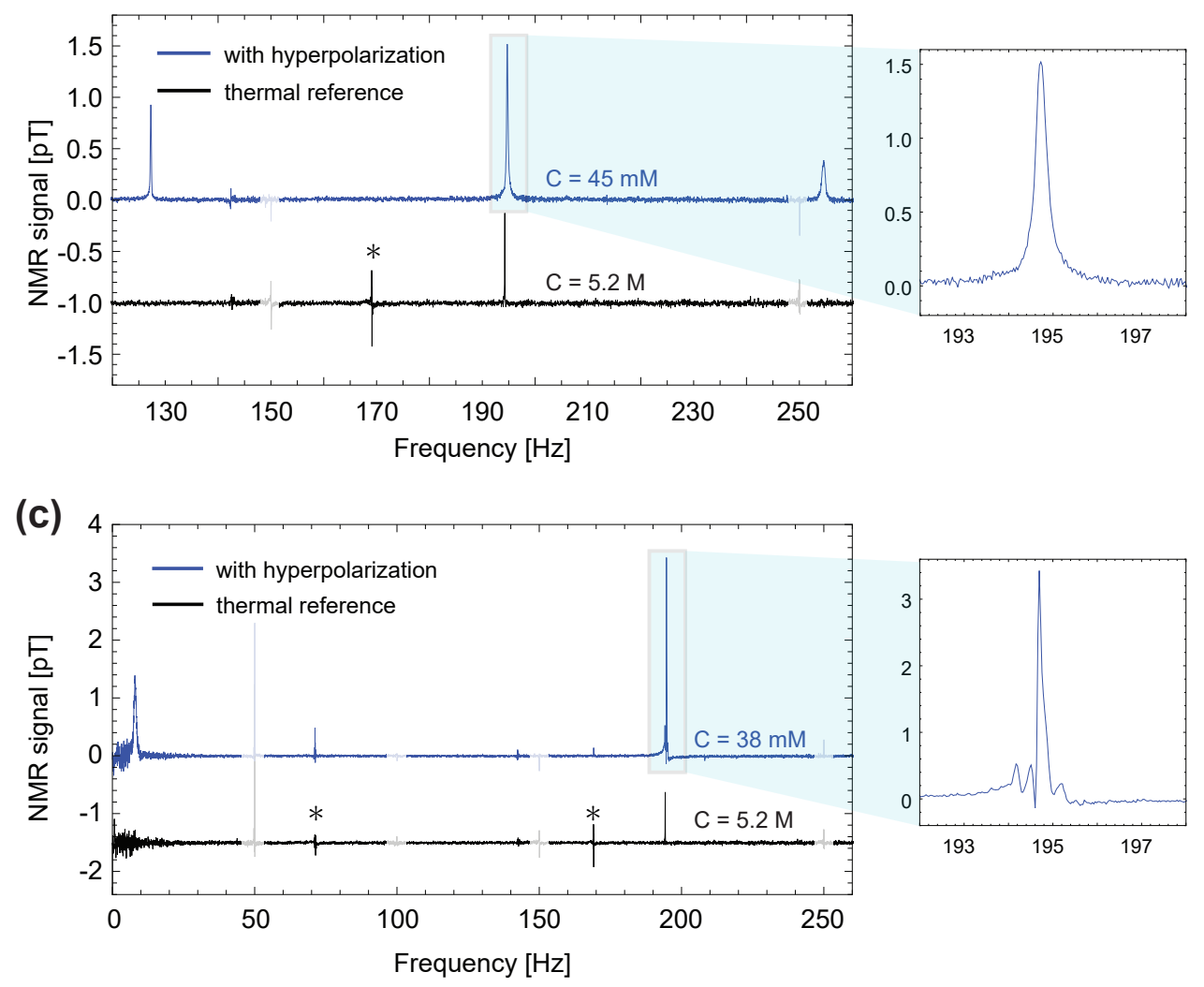

FIG. 2. ZULF spectra with $d$ DNP hyperpolarization. (a) Molecules measured in this work. (b) Single-scan ZULF spectrum of the hyperpolarized sample HP1 (blue trace) compared with a reference spectrum of $5.2 \mathrm{M}\left[{ }^{13} \mathrm{C}\right]$ sodium formate thermally prepolarized at $2 \mathrm{~T}$ after averaging sixteen scans (black trace). The thermal-reference spectrum is rescaled and offset for better visualization. (c) Single-scan ZULF spectrum of the hyperpolarized sample HP2 (blue trace) compared with the same reference spectrum as in panel (a) (black trace). The noise peaks arising from the power line at $50 \mathrm{~Hz}$ and overtones are grayed out, and probe-laser noise peaks are depicted with asterisks. The insets show the formate peak for both mixtures with the same units.

best-performing experiment yielded an enhancement of $\varepsilon=11000$, less than a quarter of the expected 51000 . Figure $3 \mathrm{~b}$ shows the signal enhancement of $d \mathrm{DNP}-\mathrm{ZULF}$ experiments with respect to prepolarization at $2 \mathrm{~T}$, calculated with Eq. 10. The result of our best-performing experiment is represented by the white line. As both ${ }^{1} \mathrm{H}$ and ${ }^{13} \mathrm{C}$ polarization contribute to the enhancement, the value of $\varepsilon=11000$ cannot be represented as a point on the plane but rather as a linear combination of ${ }^{1} \mathrm{H}$ and ${ }^{13} \mathrm{C}$ polarization. For example, if the ${ }^{1} \mathrm{H}$ polarization was $P\left[{ }^{1} \mathrm{H}\right]=0 \%$, the ${ }^{13} \mathrm{C}$ polarization of the 11000 -enhanced signal would be $P\left[{ }^{13} \mathrm{C}\right]=-6.7 \%$. The enhancement due to polarization levels achieved with a state-of-the-art $d$ DNP apparatus as reported in reference [38] is shown in Fig. 3as a long-term goal for $d$ DNP-ZULF NMR.

\section{B. Paramagnetic relaxation in $d$ DNP-ZULF NMR}

To estimate the effect of the unpaired electrons from TEMPOL on nuclear spin relaxation $[39,40]$, we performed relaxometry experiments on a sample of $5.2 \mathrm{M}$ $\left[{ }^{13} \mathrm{C}\right]$ sodium formate diluted in $\mathrm{D}_{2} \mathrm{O}$ using the zero-field spectrometer, for which we add TEMPOL in increasing concentration.

In each experiment, the sample was degassed by $\mathrm{N}_{2-}$ bubbling for $10 \mathrm{~min}$ to remove paramagnetic oxygen, which would contribute to nuclear spin relaxation. The experiments were carried out at the relevant magnetic fields used in the $d$ DNP experiments, i.e., the transfer field of $100 \mu \mathrm{T}$ and the detection field, i.e., zero field.

We studied the relaxation rate constants for coherences 
(a)
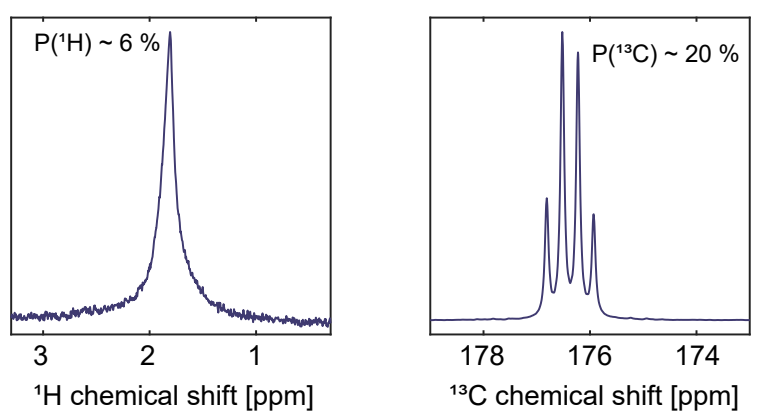

(b)

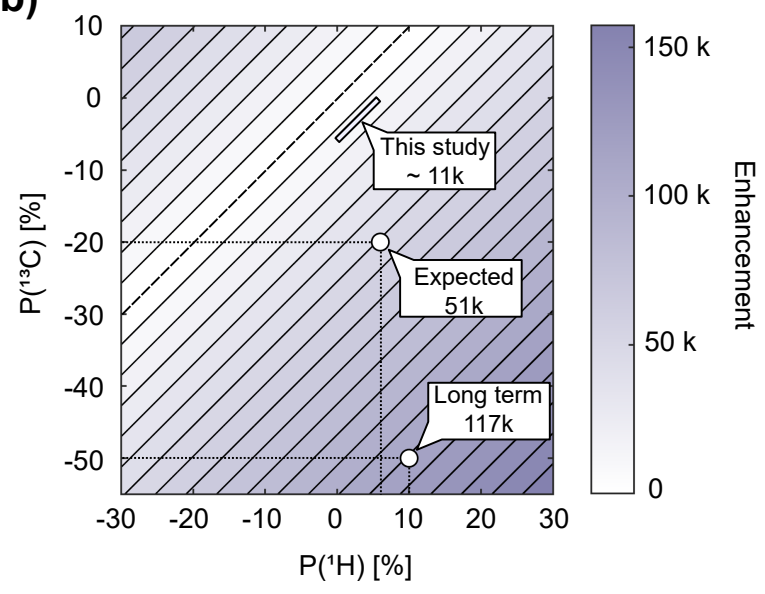

FIG. 3. (a) Typical results of $d \mathrm{DNP}$ experiments where a hyperpolarized sample of $\left[1-{ }^{13} \mathrm{C}\right]$ sodium acetate is detected at $1.88 \mathrm{~T}$ for both ${ }^{1} \mathrm{H}$ and ${ }^{13} \mathrm{C}$ channels. (b) Map of the $d \mathrm{DNP}-$ ZULF enhancement with respect to thermal polarization at $2 \mathrm{~T}$ as a function of the ${ }^{1} \mathrm{H}$ and ${ }^{13} \mathrm{C}$ polarizations calculated using Eq. 10.

$\left(1 / T_{2}\right)$, and populations $\left(1 / T_{1}\right)[36]$. The results are presented in Fig. 4. The relaxation rate constants are shown on the right as a function of TEMPOL concentration, while the pulse sequence applied in each experiment is depicted on the left. The samples were prepolarized with a $2 \mathrm{~T}$ Halbach magnet and pneumatically shuttled into the $\mu$-metal shield, where a Helmholtz coil provided a field of $100 \mu \mathrm{T}$ along the sensitive axis. This field was high enough so that the spin states remain eigenstates of the Zeeman basis. The $\left(1 / T_{1}\right)$ rate constant is determined by indirect sampling of a variable delay time $\tau$ at different fields. In Fig. 4a the sample was kept at $100 \mu \mathrm{T}$ for a variable duration before the field was rapidly switched off prior to data acquisition.

For the measurement of $\left(1 / T_{1}^{\mathrm{ZF}}\right)$ at zero field in Fig. $4 \mathrm{~b}$, the field provided by the Helmholtz coil was adiabatically brought down to zero within $50 \mathrm{~ms}$. This adiabaticity ensures that the population of the Zeeman states are converted into populations of the zero-field eigenstates. After a variable time $\tau$ at zero field, a pulse of $470 \mu \mathrm{T}$ and $100 \mu$ s duration was applied along the same axis, cor- (a)
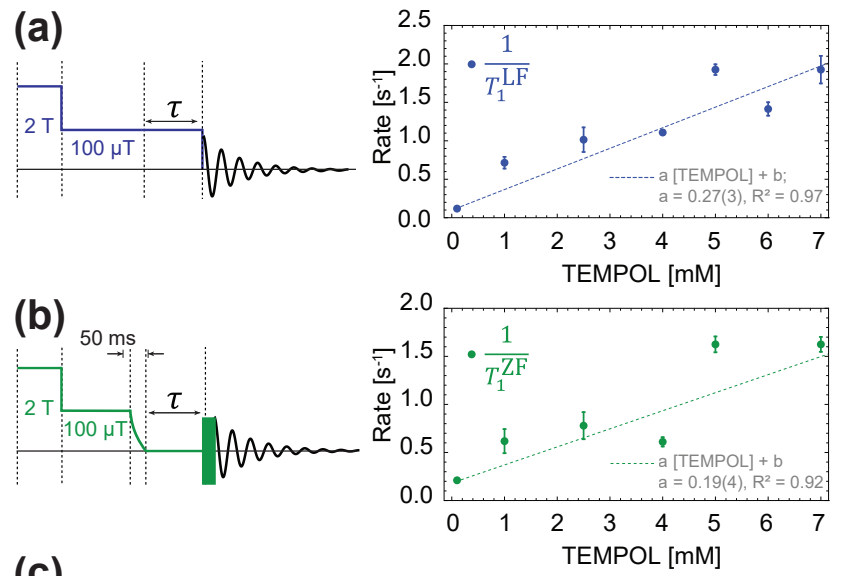

(c)

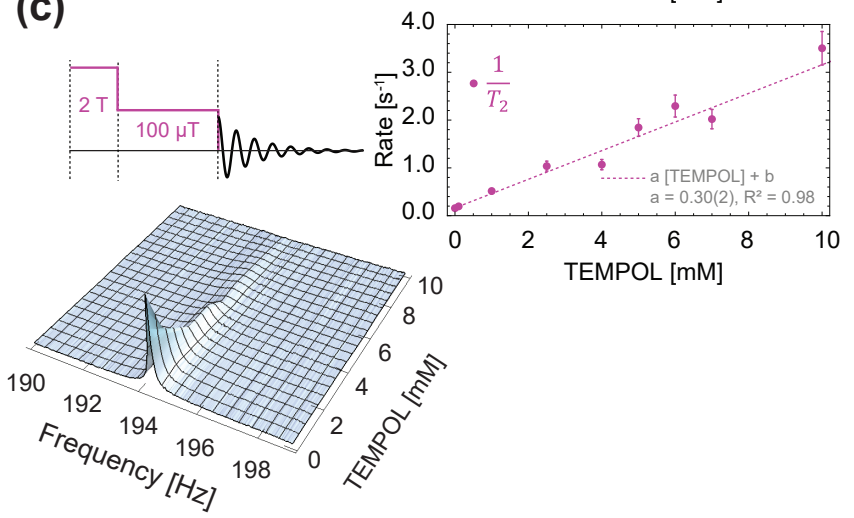

FIG. 4. Relaxation rate constants as a function of TEMPOL concentration. The sample used was $5.2 \mathrm{M}\left[{ }^{13} \mathrm{C}\right]$ sodium formate diluted in $\mathrm{D}_{2} \mathrm{O}$ degassed by $\mathrm{N}_{2}$-bubbling for $10 \mathrm{~min}$. For each sample, the broadening mechanism in the presence of free radicals was studied at $100 \mu \mathrm{T}$ and zero field. The pulse sequence applied for each experiment is shown on the left, and the relaxation constant as a function of radical concentration on the right. (a) The sample is kept at $100 \mu \mathrm{T}$ prior to rapid switch-off of the field and data acquisition. (b) The field is adiabatically switched off and a variable time is set before a $\pi$-pulse on carbon (solid block) is applied and the signal is obtained. (c) Linewidth of the NMR signal increases with TEMPOL concentration.

responding to a $\pi$-pulse on carbon. In Fig. $4 \mathrm{c}$ the NMR signals for the $T_{2}$ measurements were obtained after rapid field-drop and signal acquisition at zero field. Additionally, the NMR signal is shown under the pulse sequence as a surface, broadening with increasing radical concentration.

For all measurements presented in Fig. 4, each NMR signal was fitted with a complex Lorentzian. In the case of $\left(1 / T_{1}\right)$ measurements, the fitted values of intensity as a function of the relaxation delay $\tau$ were in turn fitted with a monoexponential function of the form $a e^{\left(t / T_{1}\right)}+b$. In the case of data taken at $100 \mu \mathrm{T}$, the signal should be more adequately fitted by a bi-exponential decay function [41], arising from the different relaxation rates of ${ }^{1} \mathrm{H}$ and ${ }^{13} \mathrm{C}$ spins. However, since protons contribute more greatly to the initial thermal polarization, mono- 
exponential fitting sufficed to fit the data with $R^{2}>0.99$. The $T_{2}$ time constant was inferred from the fitted full width at half maximum (FWHM) $\Gamma$ using the relation $\Gamma=1 /\left(\pi T_{2}\right)$.

Assuming that the paramagnetic relaxation rates are proportional to TEMPOL concentration, we fitted the curves of relaxation rate constant as a function of TEMPOL concentration with linear functions. We found that, for each relaxation constant $\left(T_{1}^{\mathrm{LF}}, T_{1}^{\mathrm{ZF}}\right.$ and $\left.T_{2}\right)$, the TEMPOL relaxivity is $a \leq 0.3 \mathrm{~s}^{-1}[\mathrm{mM}]^{-1}$, where $a$ is the fitting parameter in the inset on the right of Fig. 4 . In terms of the FWHM of the signal in presence of TEMPOL, our results show that the broadening per $\mathrm{mM}$ of TEMPOL is $\Gamma=1 /(\pi a) \approx 100 \mathrm{mHz} / \mathrm{mM}$.

\section{DISCUSSION}

The relaxometry data from Fig. 4 prove that it is possible to observe ZULF spectra of samples even in the presence of $\mathrm{mM}$ paramagnetic radical concentration, although at the price of significant paramagnetic broadening and therefore loss in resolution. The dissolution step of our $d \mathrm{DNP}$ experiments currently dilutes the sample by a factor of 30, bringing the TEMPOL concentration down to $1-2 \mathrm{mM}$. The concentration is further reduced by the presence of sodium ascorbate in the beads placed in the DNP sample holder and in the dissolution solvent, which acts as a scavenger for TEMPOL radicals [27]. However, the reaction between ascorbate and TEMPOL is not instantaneous. Due to the complex temperature and concentration dynamics of the dissolution step, it is not possible to know the concentration of TEMPOL precisely at the moment of injection. Considering that the TEMPOL concentration after injection is at most on the order of $1 \mathrm{mM}$, the results presented in Fig. 4 show that the broadening due to paramagnetic relaxation is expected to be within a few hundreds of $\mathrm{mHz}$, acceptable for our hyperpolarized experiment. The spectra of hyperpolarized samples in Fig. 2 have linewidths of approximately $200 \mathrm{mHz}$ : were this due only to TEMPOL, it would mean that $1-2 \mathrm{mM}$ of radical was present at the sample at the moment of detection, consistent with our expectations. This broadening does not decrease the signal-to-noise ratio sufficiently to prevent us from observing hyperpolarized peaks but leads to a non-negligible signal loss during the settling time $t_{\text {settle }}=2.1 \mathrm{~s}$. The remaining signal after $2.1 \mathrm{~s}$ at $100 \mu \mathrm{T}$ for a TEMPOL concentration of $2 \mathrm{mM}$ should be $\exp \left(-a\right.$ [TEMPOL] $\left.t_{\text {settle }}\right) \approx 0.28$ of the initial value. Paramagnetic relaxation during the transfer and settling of the solution could therefore explain why the recorded enhancement is lower than expected. This shows that increasing the speed of the transfer, shortening settling time, and suppressing paramagnetic relaxation is of paramount importance to improve the sensitivity of $d$ DNP-ZULF experiments. The signal-to-noise (SNR) ratio (signal amplitude divided by the standard deviation of the nearby noise range) in the
ZULF spectra in Fig. 2 is only one order of magnitude higher than a single scan of the thermal reference sample, but the key difference is the hundred-fold dilution of the biomolecules compared with the thermal polarization experiment. Furthermore, the formate peak of Fig. 2c has SNR > 500, over an order of magnitude higher as compared with previously published work [23]. Nevertheless, the polarization levels in Fig. 3 differ from the optimum performance that could be brought by the $d$ DNP apparatus by an order of magnitude. This could be due to imperfections in the magnetic tunnel leading to zerofield crossings that erode polarization, a mismatch in the synchronization of the apparatuses, or temperature and magnetic field gradients that reduce the magnetometer sensitivity. In Fig. 2 we can observe that the peak arising from glycine at $8 \mathrm{~Hz}$ is significantly broader than the other peaks of the hyperpolarized samples. It is well known that quadrupolar relaxation $[42,43]$ is significant in spin systems where nuclei with spins $>1 / 2$ are involved. The broadening is possibly due to quadrupole relaxation caused by the spin- $1{ }^{14} \mathrm{~N}$ nucleus. This is important because it implies that in the zero-field detection of $d$ DNP-polarized molecules such as amino acids and other nitrogen-containing molecules, the use of ${ }^{15} \mathrm{~N}$-labelled material may be necessary. Although $\left[1-{ }^{13} \mathrm{C}\right]$ pyruvate is polarized along with $\left[1-{ }^{13} \mathrm{C}\right]$ glycine, it is not visible in the ZULF spectrum in Fig. 2c. We believe there are several reasons, considering that the $\left[2{ }^{13} \mathrm{C}\right]$ isotopomer was observed in previous work [23]. First, the peaks are expected at lower frequencies than those of the $\left[2-{ }^{13} \mathrm{C}\right]$ isotopomer, since the ${ }^{13} \mathrm{C}$ in the first labeling position is weakly coupled to the three protons in the methyl group. As previously discussed, this $\mathrm{XA}_{3}$ group would yield peaks at ${ }^{3} J_{\mathrm{CH}}=1.3 \mathrm{~Hz}$ and $2 \times{ }^{3} J_{\mathrm{CH}}=2.6 \mathrm{~Hz}$. This poses a challenge since at very low frequencies, the magnetometer sensitivity is decreased due to $1 / f$-noise. Second, pyruvate is known to have two forms in aqueous solutions, i.e., the hydrate and non-hydrate form, which show peaks at additional frequencies, which decreases the overall signal amplitude [44]. Is it worth noting that in Fig. 2c, the formate peak shows a non-Lorentzian profile. We believe this is due to uncompensated magnetic-field gradients that hampered the shimming procedure at the sample location. This effect is also present in Fig. 2b, albeit concealed on account of a broader linewidth.

\section{CONCLUSIONS AND OUTLOOK}

We have demonstrated the feasibility of zero-field NMR detection of biomolecules hyperpolarized via dissolution dynamic nuclear polarization. Using our combined experimental setup we were able to acquire zerofield spectra of arbitrary sample mixtures thanks to the generality of $d \mathrm{DNP}$ as a hyperpolarization method, providing signal enhancements of several orders of magnitude compared with thermal prepolarization at $2 \mathrm{~T}$. We estimated the expected broadening due to paramagnetic 
relaxation of the free radicals commonly used in DNP, and found consistency with the spectra of the hyperpolarized samples.

As discussed in section $\mathrm{V}$, there is room for improvement in the SNR of the ZULF spectra by improving the sensitivity of the optically-pumped magnetometer for detection, reducing the polarization loss during sample transfer, and by increasing the concentration of hyperpolarized molecules. An appealing option to improve detection sensitivity is to employ high-sensitivity commercial OPMs, which also serve to make ZULF NMR more accessible to a wider community [45]. In order to decrease the polarization losses during sample transfer, some promising methodologies can be implemented: the use of hybrid polarizing solids (HYPSOs) and hyperpolarizing polymers (HYPOPs) avoid the presence of paramagnetic radicals in the sample after dissolution which, as discussed in section IV B, induces relaxation and subsequent signal loss [25, 46-48]. The use of HYPOP material as the polarizing medium will also increase the range of possible applications of $d$ DNP-ZULF NMR as the use of HYPOPs is independent of the solution they host. In addition, faster dissolution and injection have been demonstrated using high-pressure liquids as propellers for the dissolved sample $[24,49,50]$, allowing for dissolution and transfer in $<2 \mathrm{~s}$. The approach taken by Kouřil et al. reduces the sample-transfer duration even further (70 ms over a $3.2 \mathrm{~m}$ distance demonstrated) by pneumatically shuttling the sample in the solid state [51]. These experimental advances should further improve the performance of the $d$ DNP-ZULF experiment.

The implementation of this hyperpolarization technique for ZULF NMR opens up new avenues of investigations since $d$ DNP can be used to polarize a broad range of molecules. For example, spectral identification of dilute material can help in monitoring chemical reactions of metabolites. As discussed in the previous section, ${ }^{15} \mathrm{~N}$-labelled material may prove useful if quadrupolar relaxation is to be avoided.

A promising application of ZULF NMR is the monitoring of catalytic chemical reactions in conditions which are relevant to industry. Burueva et al have already shown an example where ZULF NMR was combined with PHIP hyperpolarization in order to monitor a chemical reaction with high resolution within a metal container and in presence of a heterogeneous catalyst [4]. However, because this approach relies on PHIP hyperpolarization, it is limited to reactions where hydrogen gas is used as a reagent. Due to its low chemical specificity, $d$ DNP-ZULF
NMR could be used for the monitoring of a wider range of chemical reactions. ZULF is a regime where chemical reactions may be monitored with high resolution for both homogeneous and heterogeneous catalysts, due to the insensitivity of ZULF to inhomogeneities induced by the magnetic susceptibility of the sample $[52,53]$. With the high sensitivity and chemical versatility offered by $d \mathrm{DNP}$, ZULF may enable the study the catalytic hydrogenation of unsaturated compounds or oligomerization and polymerization processes by high-resolution NMR [54].

Optimizing experimental parameters, leading to an overall enhancement of SNR, could also improve the search for dark matter with zero-field NMR, where exclusion parameters of axion and axion-like particles (ALPs) was obtained using a thermal polarization scheme $[6,7]$.

Our work on $d$ DNP-ZULF offers competitive proton and carbon polarization and sample concentration, as well as broader applicability of target molecules, compared to techniques using parahydrogen. We expect that $d$ DNP will become a standard choice of hyperpolarization for observing dilute nuclei in zero-field NMR, especially given the recently demonstrated possibility to polarize samples via $d$ DNP in a remote laboratory [25].

\section{ACKNOWLEDGEMENTS}

This work was supported in part by the European Research Council (ERC) under the European Union Horizon 2020 research and innovation program (grant agreement No. 695405, ERC No. 714519 / HP4all and Marie Skłodowska-Curie No. 766402 / ZULF), by the DFG via the Reinhart Koselleck project, and by the Cluster of Excellence Precision Physics, Fundamental Interactions, and Structure of Matter (PRISMA+ EXC 2118/1) funded by the DFG within the German Excellence Strategy (Project ID 39083149). The authors gratefully acknowledge Bruker Biospin for providing the prototype $d$ DNP polarizer and the F80 benchtop spectrometer for the results presented in Fig. 3, and particularly Dmitry Eshchenko, Roberto Melzi, Marc Rossire, Marco Sacher, Venita Decker, Franck Decker, and James Kempf for scientific and technical support. Catherine Jose and Christophe Pages for use of the ISA Prototype Service; and Stéphane Martinez of the UCBL mechanical workshop for machining parts of the experimental apparatus. This work was also supported by ENS-Lyon, the French CNRS, Lyon 1 University. The authors are also grateful to Lars Sperlich for helping in moving the setup from Mainz to the HMRlab in Lyon.
[1] S. Benders, M. Mohammadi, C. A. Klug, and A. Jerschow, "Nuclear magnetic resonance spectroscopy of rechargeable pouch cell batteries: beating the skin depth by excitation and detection via the casing," Scientific reports, vol. 10, no. 1, pp. 1-7, 2020.
[2] J. W. Blanchard and D. Budker, "Zero- to Ultralow-field NMR," eMagRes, vol. 5, pp. 1395-1410, 2016.

[3] J. W. Blanchard, D. Budker, and A. Trabesinger, "Lower than low: Perspectives on zero-to ultralow-field nuclear magnetic resonance," Journal of Magnetic Resonance, 
vol. 323, p. 106886, 2021.

[4] D. B. Burueva, J. Eills, J. W. Blanchard, A. Garcon, R. Picazo-Frutos, K. V. Kovtunov, I. V. Koptyug, and D. Budker, "Chemical reaction monitoring using zerofield nuclear magnetic resonance enables study of heterogeneous samples in metal containers," Angewandte Chemie International Edition, vol. 59, no. 39, pp. 1702617032, 2020.

[5] J. W. Blanchard, M. P. Ledbetter, T. Theis, M. C. Butler, D. Budker, and A. Pines, "High-resolution zero-field nmr $J$-spectroscopy of aromatic compounds," Journal of the American Chemical Society, vol. 135, no. 9, pp. 36073612, 2013.

[6] A. Garcon, J. W. Blanchard, G. P. Centers, N. L. Figueroa, P. W. Graham, D. F. J. Kimball, S. Rajendran, A. O. Sushkov, Y. V. Stadnik, A. Wickenbrock, et al., "Constraints on bosonic dark matter from ultralow-field nuclear magnetic resonance," Science advances, vol. 5, no. 10, p. eaax4539, 2019.

[7] T. Wu, J. W. Blanchard, G. P. Centers, N. L. Figueroa, A. Garcon, P. W. Graham, D. F. J. Kimball, S. Rajendran, Y. V. Stadnik, A. O. Sushkov, et al., "Search for axionlike dark matter with a liquid-state nuclear spin comagnetometer," Physical Review Letters, vol. 122, no. 19, p. 191302, 2019.

[8] M. C. Tayler, T. Theis, T. F. Sjolander, J. W. Blanchard, A. Kentner, S. Pustelny, A. Pines, and D. Budker, "Invited review article: Instrumentation for nuclear magnetic resonance in zero and ultralow magnetic field," Review of Scientific Instruments, vol. 88, no. 9, p. 091101, 2017.

[9] M. C. Tayler, J. Ward-Williams, and L. F. Gladden, "Ultralow-field nuclear magnetic resonance of liquids confined in ferromagnetic and paramagnetic materials," $A p$ plied Physics Letters, vol. 115, no. 7, p. 072409, 2019.

[10] P. Nikolaou, B. M. Goodson, and E. Y. Chekmenev, "NMR hyperpolarization techniques for biomedicine," Chemistry (Weinheim an der Bergstrasse, Germany), vol. 21, no. 8, p. 3156, 2015.

[11] K. V. Kovtunov, E. V. Pokochueva, O. G. Salnikov, S. F. Cousin, D. Kurzbach, B. Vuichoud, S. Jannin, E. Y. Chekmenev, B. M. Goodson, D. A. Barskiy, et al., "Hyperpolarized NMR spectroscopy: d-DNP, PHIP, and SABRE techniques," Chemistry-An Asian Journal, vol. 13 , no. 15 , pp. $1857-1871,2018$.

[12] O. Semenova, P. M. Richardson, A. J. Parrott, A. Nordon, M. E. Halse, and S. B. Duckett, "Reaction monitoring using sabre-hyperpolarized benchtop (1 T) NMR spectroscopy," Analytical chemistry, vol. 91, no. 10, pp. 6695-6701, 2019.

[13] T. Theis, P. Ganssle, G. Kervern, S. Knappe, J. Kitching, M. Ledbetter, D. Budker, and A. Pines, "Parahydrogenenhanced zero-field nuclear magnetic resonance," Nature Physics, vol. 7, no. 7, pp. 571-575, 2011.

[14] R. W. Adams, J. A. Aguilar, K. D. Atkinson, M. J. Cowley, P. I. Elliott, S. B. Duckett, G. G. Green, I. G. Khazal, J. López-Serrano, and D. C. Williamson, "Reversible interactions with para-hydrogen enhance NMR sensitivity by polarization transfer," Science, vol. 323, no. 5922, pp. 1708-1711, 2009.

[15] V. Yashchuk, J. Granwehr, D. Kimball, S. Rochester, A. Trabesinger, J. Urban, D. Budker, and A. Pines, "Hyperpolarized xenon nuclear spins detected by optical atomic magnetometry," Physical review letters, vol. 93, no. 16, p. 160801, 2004.

[16] R. Jiménez-Martínez, D. J. Kennedy, M. Rosenbluh, E. A. Donley, S. Knappe, S. J. Seltzer, H. L. Ring, V. S. Bajaj, and J. Kitching, "Optical hyperpolarization and NMR detection of 129 Xe on a microfluidic chip," Nature communications, vol. 5, no. 1, pp. 1-6, 2014.

[17] D. J. Kennedy, S. J. Seltzer, R. Jiménez-Martínez, H. L. Ring, N. S. Malecek, S. Knappe, E. A. Donley, J. Kitching, V. S. Bajaj, and A. Pines, "An optimized microfabricated platform for the optical generation and detection of hyperpolarized 129 Xe," Scientific reports, vol. 7, no. 1, pp. 1-10, 2017.

[18] M. P. Augustine, A. Wong-Foy, J. L. Yarger, M. Tomaselli, A. Pines, D. M. TonThat, and J. Clarke, "Low field magnetic resonance images of polarized noble gases obtained with a dc superconducting quantum interference device," Applied physics letters, vol. 72, no. 15, pp. 1908-1910, 1998.

[19] J. H. Ardenkjær-Larsen, B. Fridlund, A. Gram, G. Hansson, L. Hansson, M. H. Lerche, R. Servin, M. Thaning, and K. Golman, "Increase in signal-to-noise ratio of $>10,000$ times in liquid-state NMR," Proceedings of the National Academy of Sciences, vol. 100, no. 18, pp. 10158-10163, 2003.

[20] S. Jannin, J.-N. Dumez, P. Giraudeau, and D. Kurzbach, "Application and methodology of dissolution dynamic nuclear polarization in physical, chemical and biological contexts," Journal of Magnetic Resonance, vol. 305, pp. 41-50, 2019.

[21] B. Plainchont, P. Berruyer, J.-N. Dumez, S. Jannin, and P. Giraudeau, "Dynamic nuclear polarization opens new perspectives for $\mathrm{nmr}$ spectroscopy in analytical chemistry," 2018.

[22] S. J. Elliott, Q. Stern, M. Ceillier, T. El Daraï, S. F. Cousin, O. Cala, and S. Jannin, "Practical dissolution dynamic nuclear polarization," Progress in Nuclear Magnetic Resonance Spectroscopy, 2021.

[23] D. A. Barskiy, M. C. Tayler, I. Marco-Rius, J. Kurhanewicz, D. B. Vigneron, S. Cikrikci, A. Aydogdu, M. Reh, A. N. Pravdivtsev, J.-B. Hövener, et al., "Zero-field nuclear magnetic resonance of chemically exchanging systems," Nature communications, vol. 10, no. 1, pp. 1-9, 2019.

[24] H.-Y. Chen and C. Hilty, "Implementation and characterization of flow injection in dissolution DNP-NMR," Chemphyschem: a European journal of chemical physics and physical chemistry, vol. 16, no. 12, p. 2646, 2015.

[25] T. El Daraï, S. F. Cousin, Q. Stern, M. Ceillier, J. Kempf, D. Eshchenko, R. Melzi, M. Schnell, L. Gremillard, A. Bornet, et al., "Porous functionalized polymers enable generating and transporting hyperpolarized mixtures of metabolites," Nature Communications, vol. 12, no. 1, pp. 1-9, 2021.

[26] W. Köckenberger, "Dissolution dynamic nuclear polarization," eMagRes, pp. 161-170, 2007.

[27] P. Miéville, P. Ahuja, R. Sarkar, S. Jannin, P. R. Vasos, S. Gerber-Lemaire, M. Mishkovsky, A. Comment, R. Gruetter, O. Ouari, et al., "Scavenging free radicals to preserve enhancement and extend relaxation times in NMR using dynamic nuclear polarization," Angewandte Chemie International Edition, vol. 49, no. 35, pp. 61826185, 2010.

[28] A. Bornet, J. Milani, B. Vuichoud, A. J. P. Linde, G. Bodenhausen, and S. Jannin, "Microwave frequency mod- 
ulation to enhance dissolution dynamic nuclear polarization," Chemical Physics Letters, vol. 602, pp. 63-67, 2014.

[29] A. Bornet, R. Melzi, A. J. Perez Linde, P. Hautle, B. van den Brandt, S. Jannin, and G. Bodenhausen, "Boosting dissolution dynamic nuclear polarization by cross polarization," The journal of physical chemistry letters, vol. 4, no. 1, pp. 111-114, 2013.

[30] A. Bornet, A. Pinon, A. Jhajharia, M. Baudin, X. Ji, L. Emsley, G. Bodenhausen, J. H. Ardenkjaer-Larsen, and S. Jannin, "Microwave-gated dynamic nuclear polarization," Physical Chemistry Chemical Physics, vol. 18, no. 44, pp. 30530-30535, 2016.

[31] S. Jannin, A. Bornet, R. Melzi, and G. Bodenhausen, "High field dynamic nuclear polarization at $6.7 \mathrm{~T}$ : Carbon-13 polarization above $70 \%$ within 20 min," Chemical Physics Letters, vol. 549, pp. 99-102, 2012.

[32] A. Bornet, R. Melzi, S. Jannin, and G. Bodenhausen, "Cross polarization for dissolution dynamic nuclear polarization experiments at readily accessible temperatures $1.2<\mathrm{T}<4.2 \mathrm{~K}$," Applied Magnetic Resonance, vol. 43, no. 1, pp. 107-117, 2012.

[33] M. Jiang, R. P. Frutos, T. Wu, J. W. Blanchard, X. Peng, and D. Budker, "Magnetic gradiometer for the detection of zero-to ultralow-field nuclear magnetic resonance," Physical Review Applied, vol. 11, no. 2, p. 024005, 2019.

[34] D. Budker and M. Romalis, "Optical magnetometry," Nature physics, vol. 3, no. 4, pp. 227-234, 2007.

[35] H. J. Bernstein, J. A. Pople, and W. Schneider, "The analysis of nuclear magnetic resonance spectra: I. systems of two and three nuclei," Canadian Journal of Chemistry, vol. 35, no. 1, pp. 67-83, 1957.

[36] M. Emondts, M. P. Ledbetter, S. Pustelny, T. Theis, B. Patton, J. W. Blanchard, M. C. Butler, D. Budker, and A. Pines, "Long-lived heteronuclear spin-singlet states in liquids at a zero magnetic field," Physical review letters, vol. 112, no. 7, p. 077601, 2014.

[37] M. Ledbetter, C. Crawford, A. Pines, D. Wemmer, S. Knappe, J. Kitching, and D. Budker, "Optical detection of NMR $J$-spectra at zero magnetic field," Journal of magnetic resonance, vol. 199, no. 1, pp. 25-29, 2009.

[38] A. C. Pinon, A. Capozzi, and J. H. Ardenkjær-Larsen, "Hyperpolarization via dissolution dynamic nuclear polarization: new technological and methodological advances," Magnetic Resonance Materials in Physics, Biology and Medicine, pp. 1-19, 2020.

[39] P. Miéville, S. Jannin, and G. Bodenhausen, "Relaxometry of insensitive nuclei: optimizing dissolution dynamic nuclear polarization," Journal of Magnetic Resonance, vol. 210, no. 1, pp. 137-140, 2011.

[40] S. Bodenstedt, M. W. Mitchell, and M. C. Tayler, "Fastfield-cycling ultralow-field nuclear magnetic relaxation dispersion," Nature Communications, vol. 12, no. 1, pp. 1-8, 2021.

[41] I. V. Zhukov, A. S. Kiryutin, A. V. Yurkovskaya, Y. A. Grishin, H.-M. Vieth, and K. L. Ivanov, "Field-cycling NMR experiments in an ultra-wide magnetic field range: relaxation and coherent polarization transfer," Physical Chemistry Chemical Physics, vol. 20, no. 18, pp. 12396$12405,2018$.
[42] J. Zhu, E. Ye, V. Terskikh, and G. Wu, "Experimental verification of the theory of nuclear quadrupole relaxation in liquids over the entire range of molecular tumbling motion," The Journal of Physical Chemistry Letters, vol. 2, no. 9, pp. 1020-1023, 2011.

[43] M. C. Tayler and L. F. Gladden, "Scalar relaxation of NMR transitions at ultralow magnetic field," Journal of Magnetic Resonance, vol. 298, pp. 101-106, 2019.

[44] Y. Pocker, J. E. Meany, B. J. Nist, and C. Zadorojny, "Reversible hydration of pyruvic acid. I. equilibrium studies," The Journal of Physical Chemistry, vol. 73, no. 9, pp. 2879-2882, 1969.

[45] J. W. Blanchard, T. Wu, J. Eills, Y. Hu, and D. Budker, "Zero-to ultralow-field nuclear magnetic resonance $J$ spectroscopy with commercial atomic magnetometers," Journal of Magnetic Resonance, vol. 314, p. 106723, 2020.

[46] M. Cavaillès, A. Bornet, X. Jaurand, B. Vuichoud, D. Baudouin, M. Baudin, L. Veyre, G. Bodenhausen, J.-N. Dumez, S. Jannin, et al., "Tailored microstructured hyperpolarizing matrices for optimal magnetic resonance imaging," Angewandte Chemie, vol. 130, no. 25, pp. $7575-7579,2018$.

[47] D. Gajan, A. Bornet, B. Vuichoud, J. Milani, R. Melzi, H. A. Van Kalkeren, L. Veyre, C. Thieuleux, M. P. Conley, W. R. Grüning, et al., "Hybrid polarizing solids for pure hyperpolarized liquids through dissolution dynamic nuclear polarization," Proceedings of the National Academy of Sciences, vol. 111, no. 41, pp. 14693-14697, 2014.

[48] D. Baudouin, H. A. Van Kalkeren, A. Bornet, B. Vuichoud, L. Veyre, M. Cavaillès, M. Schwarzwälder, W.-C. Liao, D. Gajan, G. Bodenhausen, et al., "Cubic threedimensional hybrid silica solids for nuclear hyperpolarization," Chemical science, vol. 7, no. 11, pp. 6846-6850, 2016.

[49] S. Bowen and C. Hilty, "Rapid sample injection for hyperpolarized NMR spectroscopy," Physical Chemistry Chemical Physics, vol. 12, no. 22, pp. 5766-5770, 2010.

[50] M. Ceillier, O. Cala, T. E. Daraï, S. F. Cousin, Q. Stern, S. Guibert, S. J. Elliott, A. Bornet, B. Vuichoud, J. Milani, C. Pages, D. Eshchenko, J. G. Kempf, C. Jose, S. A. Lambert, and S. Jannin, "An automated system for fast transfer and injection of hyperpolarized solutions," Journal of Magnetic Resonance Open, p. 100017, 2021.

[51] K. Kouřil, H. Kouřilová, S. Bartram, M. H. Levitt, and B. Meier, "Scalable dissolution-dynamic nuclear polarization with rapid transfer of a polarized solid," Nature communications, vol. 10, no. 1, pp. 1-6, 2019.

[52] M. Leutzsch, A. J. Sederman, L. F. Gladden, and M. D. Mantle, "In situ reaction monitoring in heterogeneous catalysts by a benchtop NMR spectrometer," Magnetic resonance imaging, vol. 56, pp. 138-143, 2019.

[53] M. C. Tayler, J. Ward-Williams, and L. F. Gladden, "NMR relaxation in porous materials at zero and ultralow magnetic fields," Journal of Magnetic Resonance, vol. 297, pp. 1-8, 2018.

[54] P. A. Boeg, J. Ø. Duus, J. H. Ardenkjær-Larsen, M. Karlsson, and S. Mossin, "Real-time detection of intermediates in rhodium-catalyzed hydrogenation of alkynes and alkenes by dissolution dnp," The Journal of Physical Chemistry C, vol. 123, no. 15, pp. 9949-9956, 2019 . 